EPJ Web of Conferences 45, 01095 (2013)

DOI: $10.1051 /$ epjconf/20134501095

(C) Owned by the authors, published by EDP Sciences, 2013

\title{
Modification of shape oscillations of an attached bubble by surfactants
}

\author{
L. Vobecká ${ }^{1, a}$, J. Vejražka ${ }^{1}$ and J. Tihon ${ }^{1}$ \\ ${ }^{1}$ Academy of Sciences of the Czech Republic, Institute of Chemical Process Fundamentals, Rozvojová 135, 16502 \\ Prague 6, Czech Republic, ${ }^{\text {a }}$ vobecka@icpf.cas.cz
}

\begin{abstract}
Surface-active agents (surfactants, e.g. washing agents) strongly modifies properties of gas-liquid interface. We have carried out extensive experiments, in which we study effect of surfactants on the shape oscillations of a bubble, which is attached at a tip of a capillary. In the experiments, shape oscillations of a bubble are invoked by a motion of a capillary, to which the bubble is injected. Decaying oscillations are recorded and their frequency and damping are evaluated. By changing the excitation frequency, three lowest oscillation modes are studied. Experiments were repeated in aqueous solution of several surfactants (terpineol, SDS, CTAB, Triton X-100, Triton X-45) at various concentrations. Generally, these features are observed: Initially a surfactant addition leads to an increase of the oscillation frequency (though surface tension is decreasing); this effect can be attributed to the increasing interfacial elasticity. The decay time of oscillation is strongly decreasing, as a consequence of energy dissipation linked with Marangoni stresses. At a certain critical concentration, frequency decreases abruptly and the decay time passes by a minimum. With further addition of surfactant, frequency decreases, and the decay time slightly lengthens. Above critical micelle concentration, all these parameters stabilize. Interestingly, the critical concentration, at which frequency drop occurs, depends on mode order. This clearly shows that the frequency drop and minimum decay time are not a consequence of some abrupt change of interfacial properties, but are a consequence of some phenomena, which still need to be explained.
\end{abstract}

\section{Introduction}

A presence of surface active agents (surfactants) can strongly modify the dynamics of multiphase systems. The impact of a bubble on a horizontal solid in aqueous solution of surfactants was recently studied [1 and 2]. It was found that after an addition of small amount of surfactants, the bubble rebound from horizontal wall is completely suppressed. The difference between the bubbles colliding in the pure water and in the aqueous terpineol solution is shown in the figure 1 .

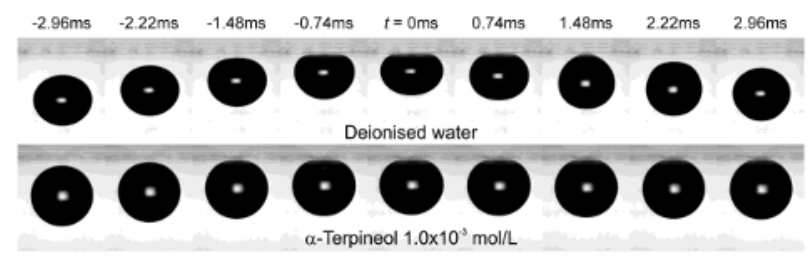

Fig. 1. The impact of a $1 \mathrm{~mm}$ bubble in pure water and in aqueous terpineol solution.

More detailed analysis suggests that the rebound suppression is linked with deformation of an interface. Interface deformations seem to dissipate energy much faster, if the interface is occupied by surfactants. To verify this hypothesis, we study bubble oscillations in the surfactant solution. During a period of bubble oscillation, the deformation has similar character as during the rebound. The energy dissipation is studied more easily from the decay of oscillations.

\section{Experiment and evaluation of bubble oscillations}

The bubble oscillations are studied in an experimental setup, which is schematized in figure 2. The setup consists of a vessel made of glass and stainless steel. A bubble grows slowly on a moveable glass capillary and its size is controlled by proper timing of filling and drainage valves. Bubble oscillations are then induced by a motion of the capillary, which is attached to an electromagnetic coil [3].

The capillary tip moves first sinusoidally with frequency adjusted close to an eigenmode frequency. This motion is maintained typically for a few hundredths of periods to achieve steady-state oscillations. The motion of the capillary tip is then progressively stopped and decaying bubble oscillations are recorded. 
The working liquids are pure water and aqueous solutions of a surfactants $\alpha$-terpineol, Triton X-45, Triton X-100, sodium dodecyl sulfate and hexadecyl trimethyl ammonium bromide, which is caled CTAB.

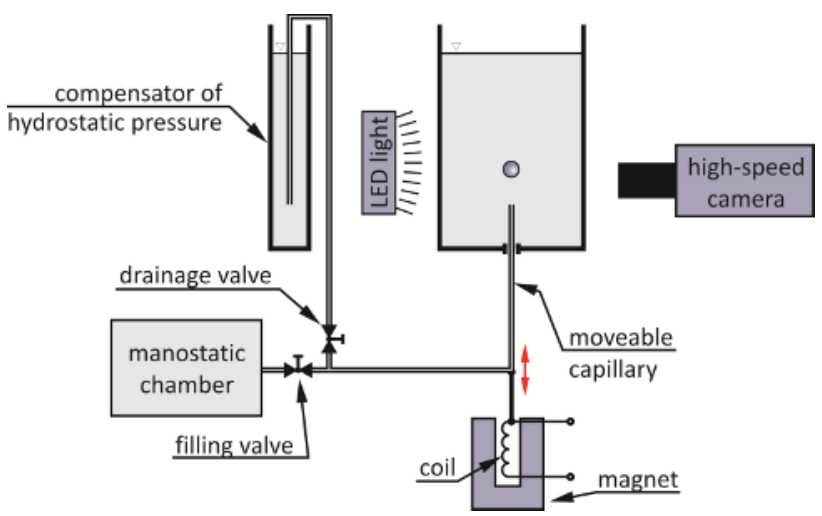

Fig. 2. Experimental setup

The bubble oscillations are recorded by a high-speed camera (Photron SA1.1); a typical movie has resolution $700 \times 320$ pixels and it is recorded with the frame rate of $18000 \mathrm{fps}$. The typical images of certain eigen-modes are shown in figure 3 . The first image of each mode shows the shape of the bubble with negative amplitude and the second image represents positive amplitude. Different eigenmodes are induced by changing the excitation frequency.
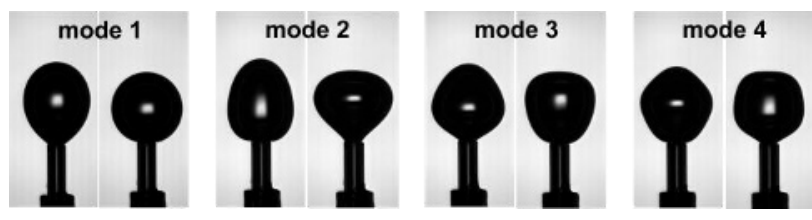

Fig. 3. Images of bubble $\left(D_{e q} \approx 1.3 \mathrm{~mm}\right)$ eigen-modes with positive and negative amplitude

The obtained sequence of image is processed by digital processing of images. The bubble boundary is found and its shape is expressed as a function $r(\theta, t)$ (see figure 4). We decompose this shape in

$$
r(\theta, t)=\sum_{k=0}^{N} A_{k}(t) P_{k}(\cos \theta)
$$

In the last equation, $P_{k}$ are Legendre polynomials and $P_{k}(\cos \theta)$ are the eigen-shapes of oscillations of a rising bubble or drop, following the classical theory of Rayleigh and Lamb [4]. Coefficients $A_{k}(t)$ are found by fitting the shape given by equation (1) to an experimentally observed shape. Coefficient $A_{0}$ describes the bubble size. The central point is chosen in the way that $A_{l}=0$; the bubble position is then given by the position of central point $z_{c p}$. Set of $A_{2}$ to $A_{N}$ describes the bubble shape; typically $N$ ranging from 8 to 15 is used and the bubble shape is then reproduced with deviation smaller than 0.3 pix.

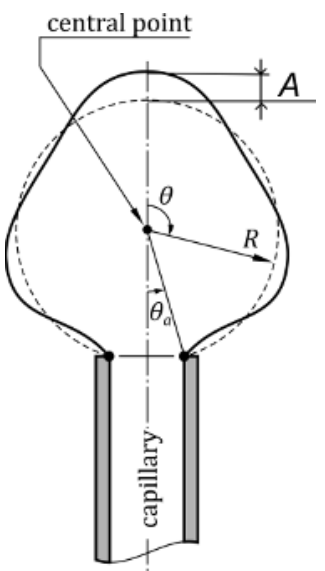

Fig. 4. Description of bubble shape

\section{Results and discussion}

\subsection{Bubble oscillations in a-terpineol}

The damping time of oscillations is strongly affected by a presence of surfactants. First, bubble oscillations were studied in detail in nonionic surfactant $\alpha$-terpineol. Then results for $\alpha$-terpineol were compared with results for different surfactants. Figure 5 shows the influence of surfactant gradual adding on dimensionless damping for three lowest egein-modes.

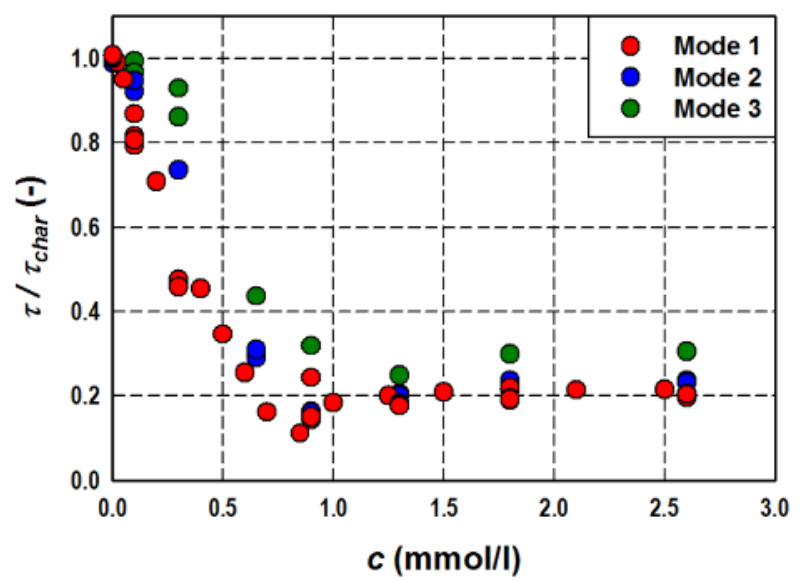

Fig. 5. Damping time in $\alpha$-terpineol solution for mode1 to 3 
The damping time is normalized by characteristic time

$$
\tau_{\text {char }}=\frac{\rho D^{2}}{\mu},
$$

and finally it is normalized by damping time in water, to start at value 1 .

Initially, the damping time decreases almost linearly with concentration, then it passes through a minimum occurring at concentration of about $0.8 \mathrm{mmol} / \mathrm{l}$ and finally it slightly increases again.

It is interesting to examine also on the oscillation frequency. The frequency dependence on surfactant is shown in figure 6 for three lowest egeinmodes. The frequency is normalized by

$$
f_{\text {char }}=\sqrt{\frac{D_{\text {att }}}{D} \sqrt{\frac{\sigma_{\text {pure }}}{\rho D^{3}}}}
$$

and again, frequency is normalized by frequency received in pure water.

In pure systems, the decrease of surface tension leads to a decrease of oscillation frequency. It is observed that the frequency initially increases, although the addition of surfactant decreases the surface tension. A decrease computed from the reduction of surface tension by surfactant is shown by blue line. This frequency increase is perhaps due to an elasticity of interface with adsorbed surfactant. There is a sudden drop of the frequency which occurs at the same concentration as the minimum of damping time. We do not have definite explanation for this frequency drop.

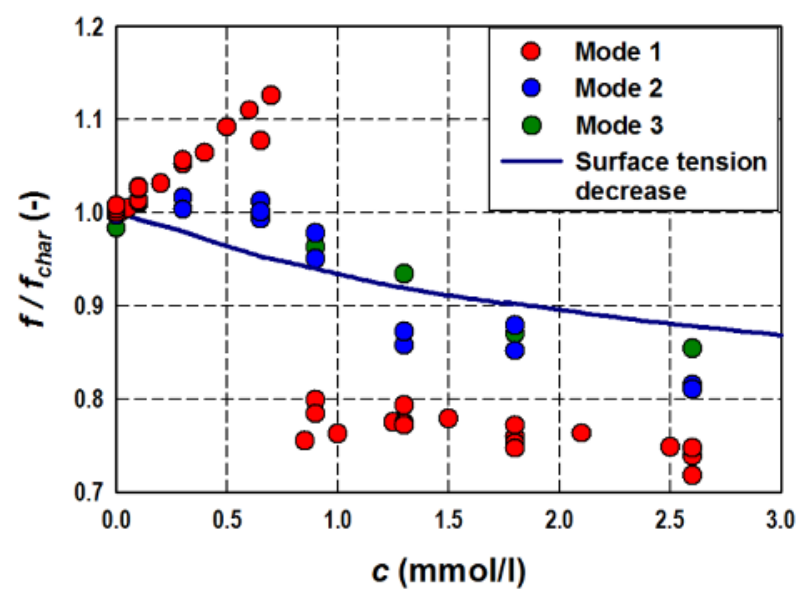

Fig. 6. Oscillation frequency in $\alpha$-terpineol solution for mode1 to 3

\subsection{Bubble oscillations in triton X-45}

To verify, if this behavior isn't specific only to the used surfactant, terpineol, experiments were repeated with other types of surfactant. Figure 7 shows the dependence of damping time on concentration of Triton X-45. Similar behavior of damping time is observed in terpineol as well as in triton X-45 solution. Damping time initially increases and then passes through a minimum. But this become at different concentration for each eigenmode.

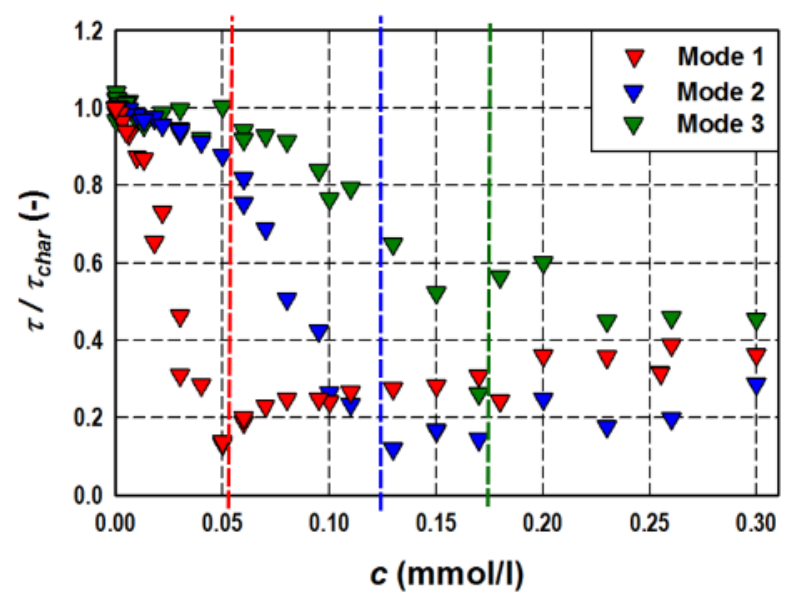

Fig. 7. Damping time in triton $\mathrm{X}-45$ solution for mode 1 to 3

The different minimum of damping time for each eigen-mode is shown by dashed lines of various colors. Red dashed line marks minimum of first eigen-mode, blue line is for second eigen-mode and green line for third eigen-mode.

The oscillation frequency of triton $\mathrm{X}-45$ is shown on figure 8 in the dependence on its concentration. Frequencies are also similar to those seen with terpineol. Frequency drops is again observed. For each mode, they occur at different concentration, which is shown by dashed line of different colors. We can say that these drops are not caused by some sudden change of physicochemical properties of surfactant solution.

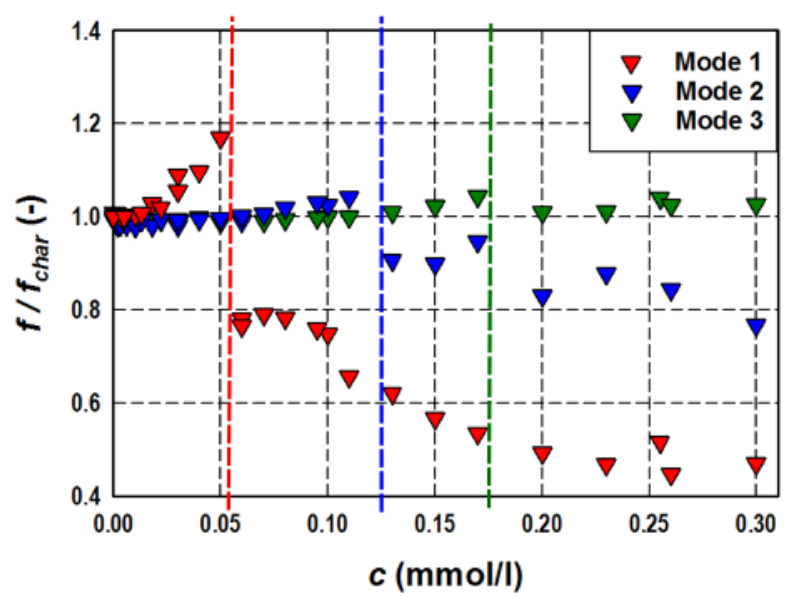

Fig. 8. Oscillation frequency in triton $\mathrm{X}-45$ solution for mode1 to 3 
Experiments were made also with other types of surfactant. The next used surfactants were triton X-100, sodium dodecyl sulphate (SDS) and hexadecyltrimethylammonium bromide (CTAB). The behavior of these surfactants is similar to $\alpha$-terpineol and triton X-45. Small concentrations of triton X-100, SDS and $\mathrm{CTAB}$ also cause rapid decrease of damping time, which passes through a minimum and then increases again. In case of triton $\mathrm{X}-100$ and $\mathrm{CTAB}$, the damping time is constant after the concentration reaches value 0,3 mmol/1 for triton X-100 and $1 \mathrm{mmnol} / \mathrm{l}$ for CTAB, which are critical micelar concentrations of these surfactants. The critical micelar concentration was not exceeded during experiments in case of $\alpha$-terpineol, triton $\mathrm{X}-45$ and SDS.

Although the surface tension decreases, the oscillation frequency initially increases in case of all used surfactants. The unexpected drop of oscillation frequency is again present for all used surfactants and the oscillation frequency remains constant after reaching critical micellar for triton X-100 and CTAB.

\section{Summary}

Bubble oscillations of a bubble attached to a capillary tip are studied in both, pure water and surfactant solutions of different types of surfactant ( $\alpha$-terpineol, triton X-45, triton $\mathrm{X}-100$, SDS and $\mathrm{CTAB}$ ). It was observed, that surfactants have strong effect on oscillations. Surfactant presence leads to rapid decrease of damping time, which rapidly increases and then slightly increases again. It is possible to conclude, that this behavior is not property only of the first detailed studied surfactant ( $\alpha$-terpineol), but also another types of surfactant.

Further, it was studied oscillation frequency for modes 1 to 3 . It was found, that unexpected drop of frequency becomes at different concentration for each eigenmode. This shows, that sudden drop is not caused by sudden change of physic-chemical properties of surfactant solution.

\section{References}

1. K. Malysa, M. Krasowska, M. Krzan, Adv. Coll. Interface Sci., 114, 205-225 (2005)

2. M. Fujasová-Zedníková, L. Vobecká, J. Vejrazka, Canadian Journal of Chemical Engineering, 88, 473$481(2010)$

3. J. Vejrazka., M. Fujasova, P. Stanovsky., M. C. Ruzicka, J. Drahos, Fluid Dyn. Res., 40, 521-533 (2008)

4. H. Lamb, Hydrodynamics, Dover, 1945 